\title{
QCD at finite temperature and density from the Curci-Ferrari model
}

\author{
Urko Reinosa ${ }^{* \dagger}$ \\ Centre de Physique Théorique, CNRS, Ecole polytechnique, IP Paris, F-91128 Palaiseau, France \\ E-mail: urko.reinosa@polytechnique.edu
}

\begin{abstract}
We summarize recent progress in describing fundamental properties of $Q C D$ at finite temperature and finite (baryonic) chemical potential using the Curci-Ferrari model, a proposal for gauge-fixing in the Landau gauge beyond the standard Faddeev-Popov construction.
\end{abstract}

Light Cone 2019 - QCD on the light cone: from hadrons to heavy ions - LC2019

16-20 September 2019

Ecole Polytechnique, Palaiseau, France

\footnotetext{
* Speaker.

${ }^{\dagger}$ Based on various works in collaboration with J. Maelger, M. Peláez, J. Serreau, M. Tissier and N. Wschebor.
} 
Although it makes no doubt that Quantum Chromodynamics (QCD) is a strongly interacting theory in the infrared, the corresponding qualification of its companion Yang-Mills (YM) theory is more open to debate. In recent years, lattice simulations in the Landau gauge have demonstrated that the renormalized YM coupling $g$ remains bounded, and even decreases towards zero in the infrared [1], in the so-called Taylor scheme. When translated in terms of the relevant perturbative expansion parameter $\lambda \equiv N g^{2} /\left(16 \pi^{2}\right)$, the bound becomes $\lambda \lesssim 0.12$ for $N=3$ colors. This has led to the idea that some sort of perturbation theory should be applicable to YM theory in the infrared [2], at least for the determination of certain quantities.

It is of course not an easy question how to set up such a perturbative expansion (were it to make sense in the first place). The traditional Faddeev-Popov (FP) gauge-fixing method is clearly of no use for this venture because it neglects the Gribov copies that are present in the Landau gauge and whose effect is a priori not negligible in the infrared. Attempts to go beyond FP include the celebrated Gribov-Zwanziger (GZ) paradigm [3] that deals with a subclass of the Gribov copies. Another possible strategy, more phenomenological in spirit, is to model the missing terms beyond the FP action using some of the salient features of Landau gauge-fixed YM theory that are now well established thanks to lattice simulations.

One such feature is the decoupling type behavior of the two-point correlation functions in the infrared [4]: while the ghost propagator behaves essentially like its free counterpart, the gluon propagator saturates to a finite non-zero value. Based on these features, a natural model for the Landau gauge-fixed action beyond FP is the so-called Curci-Ferrari (CF) action

$$
S_{\mathrm{CF}}=\int_{x}\left\{\frac{1}{4} F_{\mu \nu}^{a} F_{\mu \nu}^{a}+i h^{a} \partial_{\mu} A_{\mu}^{a}+\bar{c}^{a} \partial_{\mu} D_{\mu} c^{a}+\frac{1}{2} m^{2} A_{\mu}^{a} A_{\mu}^{a}\right\}
$$

that adds a gluon mass term to the FP action. Needless to mention that this is of course just a model and other operators could be needed in order to make the gauge-fixing complete. One convenient feature of (1) is, however, that it is stable under the renormalization flow. Therefore, there is only one new parameter to be fixed, $m$, and, in the absence of any ab-initio determination (that a bonafide gauge-fixing would provide), it is possible to fix it by comparison to the above mentioned lattice results. One finds a typical value for $m$ or the order of $500 \mathrm{MeV}$ for $N=3$ colors. The Curci-Ferrari model has been quite successful in reproducing vacuum properties of Yang-Mills correlation functions, already at one-loop order [2, 5]. Recent two-loop calculations [6] show that this is not accidental and the perturbative expansion seems well under control in the model while capturing essential features of YM theory in the Landau gauge.

Irrespectively of how interesting these observations might be, it remains to be seen how useful there are for QCD. In this case, in addition to the coupling in the pure gauge sector, one can extract a coupling $\tilde{g}$ from the matter sector. It turns out that this coupling is 2 to 3 times larger than the pure gauge coupling $g$ [7], which implies an expansion parameter $\tilde{\lambda} \equiv N \tilde{g}^{2} /\left(16 \pi^{2}\right)$, typically 5 to 10 times larger than $\lambda$ (that is $\tilde{\lambda} \gtrsim 1$ ). Of course, this is precisely the reason why QCD is strongly interacting and perturbation theory is of no use in the infrared in this case. Interestingly enough, however, the separation of couplings $\lambda \ll 1 \lesssim \tilde{\lambda}$ allows to envisage a systematic computational scheme where one expands in powers of $\lambda$ without expanding in $\tilde{\lambda}$. In [8], we have taken advantage of this observation and combined it with the $1 / N$ expansion in order to construct a systematic expansion for Landau gauge QCD that is controlled by two small parameters $(1 / N$ and $\lambda)$ and that 
is tractable at least in its leading orders. Within this approach, and already at leading order, we have been able to capture the physics of chiral symmetry breaking one of the key characteristics of the QCD vacuum. We refer to the contribution of Julien Serreau in these proceedings for more details.

Next to these considerations in the vacuum, the other pressing question is whether our approach allows to capture the phase structure of QCD (or YM theory) as a function of various control parameters such as the temperature, chemical potential, magnetic fields, ... Below, we review the progress that has been made in this direction within the CF model.

\section{Results in the YM case}

It is not completely straightforward to extend the Curci-Ferrari model to finite temperature. The reason has little to do with the Curci-Ferrari model itself and has more to do with the fact that the Landau gauge might not be a good gauge at finite temperature. ${ }^{1}$ One heuristic way to convince oneself of this fact is to consider the order parameter for the confinement/deconfinement transition in YM theory, the so-called Polyakov loop ( $\mathscr{T}$ denotes the time-ordering along the Euclidean time interval $[0, \beta=1 / T]$ )

$$
\ell \equiv \frac{1}{N}\left\langle\mathscr{T} \exp \left\{i g \int_{0}^{\beta} d \tau A_{0}^{a}(\tau, \vec{x}) t^{a}\right\}\right\rangle
$$

and to envisage evaluating it in a mean field fashion as $\ell \approx \exp \left\{\operatorname{ig} \beta\left\langle A_{0}^{a}\right\rangle t^{a}\right\}$. Since the Landau gauge has no preferred color direction, $\left\langle A_{0}^{a}\right\rangle=0$ and the mean field Polyakov loop saturates to 1, which means that it cannot be used as an order parameter, at least not at this level of approximation.

The way out is to slightly generalize the Landau gauge in the presence of an external background configuration $\bar{A}_{\mu}^{a}$ [9]. The Landau gauge condition $0=\partial_{\mu} A_{\mu}^{a}$ is replaced by the so-called Landau-deWitt condition $0=\bar{D}_{\mu}\left(A_{\mu}^{a}-\bar{A}_{\mu}^{a}\right)$ where $\bar{D}_{\mu}$ denotes the covariant derivative in the presence of the background. The background could be chosen arbitrarily a priori. In practice, however, it is chosen to be self-consistent, that is such that $\left\langle A_{\mu}^{a}\right\rangle=\bar{A}_{\mu}^{a}$. These particular backgrounds can be obtained from the minimization of the so-called background field effective action [9, 10]. More importantly, they can be shown to be alternative order parameters for the confinement/deconfinement transition $[10,11,12]$. This is easily seen at mean field level where the relation to the Polyakov loop is simply $\ell \approx \exp \left\{\operatorname{ig} \beta \bar{A}_{0}^{a} t^{a}\right\}$, so that the vanishing of $\ell$ corresponds to $\bar{A}_{0}^{a} t^{a}$ taken specific values in the algebra of the gauge group.

Just like the Landau gauge, the Landau-deWitt gauge is hampered by the presence of Gribov copies and the FP action needs to be replaced by something else. The natural extension of the model (1) in the presence of a background is

$$
S_{\mathrm{bgCF}}=\int_{x}\left\{\frac{1}{4} F_{\mu \nu}^{a} F_{\mu \nu}^{a}+i h^{a} D_{\mu}\left(A_{\mu}^{a}-\bar{A}_{\mu}^{a}\right)+\bar{c}^{a} \bar{D}_{\mu} D_{\mu} c^{a}+\frac{1}{2} m^{2}\left(A_{\mu}^{a}-\bar{A}_{\mu}^{a}\right)\left(A_{\mu}^{a}-\bar{A}_{\mu}^{a}\right)\right\} .
$$

The reason for introducing a mass for $A_{\mu}^{a}-\bar{A}_{\mu}^{a}$ rather than $A_{\mu}^{a}$ is to preserve the background gauge symmetry, one of the cornerstones of the background field formalism.

\footnotetext{
${ }^{1}$ Of course, at an exact level, all gauges should be equivalent. In practice, however, one is always bound to approximations, and in this case, certain gauges might be more efficient than others in capturing certain features.
} 
In Table 1, we summarize some of the results for the confinement/deconfinement transition, as obtained from the background extended Curci-Ferrari model (1.2) and compared to other approaches. We find a second order transition in the case of the SU(2) gauge group, and a first order transition in the case of SU(3), in agreement with numerical simulations on the lattice. Our predictions for the deconfinement temperature at one-loop order are quite reasonable given the simplicity of the approach. Moreover, the inclusion of higher order corrections considerably improves the comparison to other approaches, illustrating once more the apparent convergence of perturbation theory within the CF model.

\begin{tabular}{|c||c||c||c||c||c|}
\hline$T_{\mathrm{c}}(\mathrm{MeV})$ & lattice & fRG [13] & variational [14] & CF1 & CF2 \\
\hline \hline $\mathrm{SU}(2)$ & 295 & 230 & 239 & 238 & 284 \\
$\mathrm{SU}(3)$ & 270 & 275 & 245 & 185 & 254 \\
\hline
\end{tabular}

Table 1: Confinement/deconfinement transition temperatures as obtained from the Curci-Ferrari model at one-loop (CF1) and two-loop (CF2) order, compared to other approaches including lattice simulations.

\section{Results for heavy-quark QCD}

With not to much effort, it is possible to include quarks and a baryonic chemical potential $\mu$ :

$$
\delta S=\int_{x} \sum_{f=1}^{N_{f}} \bar{\psi}_{f}\left(\not D+M_{f}+\mu \gamma_{0}\right) \psi_{f}
$$

first in the regime where they are all consider heavy $\left(\beta M_{f} \gg 1\right)$. There are many reason for considering such a formal regime. First, we are close enough to the YM case for the phase structure to be dominated by the confinement/deconfinement transition, without the need to consider the physics of chiral symmetry breaking. Second, there is a very rich phase structure: at vanishing chemical potential, there exists a boundary line in the $\left(M_{u}=M_{d}, M_{s}\right)$ plane that separates a crossover regime from first order type transitions. The boundary line moves when varying the baryonic chemical potential and even extends to imaginary chemical potential values where it connects with the physics of the Roberge-Weiss transition (at $\beta \mu=i \pi / 3$ ).

The tables in 2 summarize some of the results obtained in the heavy quark regime using the CF model (1.2) combined with (2.1). Assuming $N_{f}=1,2$ or 3 degenerate flavours of equal mass $M$, we show the values of $R_{N_{f}} \equiv \beta M$ on the boundary line at $\mu=0$ and $\beta \mu=i \pi / 3$. We see once again that the agreement with the lattice results is pretty good. It is worth noticing that our one-loop predictions do not really depend on the precise value of $m$ but only on the existence of a non-zero $m$. We mention also that the comparison of our two-loop results with the lattice results is a bit tricky since the mass quoted by the lattice is the bare mass, while in our case it is a one-loop renormalized mass. However, it is possible to argue that, at two-loop order, the scheme dependences drop when considering ratios of $R_{N_{f}}$ at two-different values of $N_{f}$. When these ratios are considered our results show again an apparent convergence towards the lattice results. 


\begin{tabular}{|c|ccc||cc|}
\hline$\mu=0$ & $R_{1}$ & $R_{2}$ & $R_{3}$ & $R_{2} / R_{1}$ & $R_{3} / R_{2}$ \\
\hline \hline Lattice [15] & 7.23 & 7.92 & 8.33 & 1.10 & 1.05 \\
\hline \hline Matrix [16] & 8.04 & 8.85 & 9.33 & 1.10 & 1.05 \\
\hline CF1 [17] & 6.74 & 7.59 & 8.07 & 1.12 & 1.06 \\
\hline \hline CF2 [18] & 7.53 & 8.40 & 8.90 & 1.11 & 1.06 \\
\hline
\end{tabular}

\begin{tabular}{|c|ccc||cc|}
\hline$\beta \mu=i \pi / 3$ & $R_{1}$ & $R_{2}$ & $R_{3}$ & $R_{2} / R_{1}$ & $R_{3} / R_{2}$ \\
\hline \hline Lattice [15] & 5.56 & 6.25 & 6.66 & 1.12 & 1.07 \\
\hline \hline Matrix [19] & 5.00 & 5.90 & 6.40 & 1.18 & 1.08 \\
\hline CF1 [17] & 4.72 & 5.63 & 6.14 & 1.19 & 1.09 \\
\hline \hline CF2 [18] & 5.47 & 6.41 & 6.94 & 1.17 & 1.08 \\
\hline
\end{tabular}

Table 2: $R_{N_{f}}$ for $N_{f}=1,2$, and 3 degenerate quark flavors, as computed in various approaches, for $\mu=0$ and $\beta \mu=i \pi / 3$. CF1 and CF2 refer to the one- and two-loop results within the CF model.

\section{Results for QCD with light quarks}

As already mentioned in the introduction, in the case of light quarks, perturbation theory does not make any sense. We can however exploit the perturbative nature of the pure gauge sector in order to construct a systematic expansion, controlled by two small parameters, the inverse number of colors, and the coupling in the pure gauge sector. At leading order, this expansion leads to the well known rainbow equation for the quark propagator, with however specific choices for the quark-gluon vertex and gluon propagator that remain at tree-level. One benefit of our approach is that higher order corrections are small, and in fact can be included systematically, in particular in the form of renormalization group effects.

We have implemented this program entirely in the vacuum. At finite temperature and density, so far, we have solved the rainbow equation using some drastic approximations, that we believe nonetheless to capture the main qualitative features [20]. In particular, in the chiral limit, we find the phase diagram represented in Fig. 1. The red dot represents a tricritical point that becomes a critical end point as non-vanishing quark masses are considered. We note that the absence of a first order transition at $\mu=0$ seems incompatible with general arguments [21]. However, this is compatible with observations on the lattice [22] according to which, the region of first order transitions at $\mu=0$ should be small. Since corrections to our leading order calculation are controlled by two small parameters, we expect equally this region to be small (were it to exist).

In conclusion, all these results gives us good confidence that the Curci-Ferrari model is a good starting point to investigate low energy features of QCD, either perturbatively or within a systematic expansion controlled by two small parameters.

\section{References}

[1] I. L. Bogolubsky, E. M. Ilgenfritz, M. Müller-Preussker and A. Sternbeck, Phys. Lett. B 676 (2009) 69. 


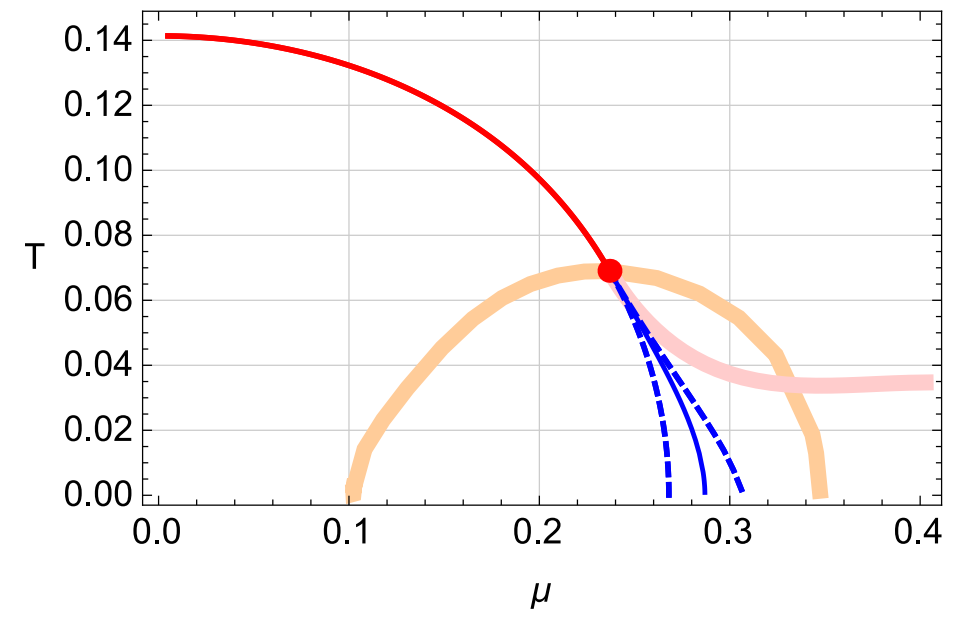

Figure 1: Phase diagram in the chiral limit (all scales in $\mathrm{GeV}$ ) as predicted from the $\mathrm{CF}$ model. Blue curve: first order transitions flanked by spinodals (dashed curves); Red curve: second order transition (becomes a crossover away from the chiral limit); Red dot: tricritical point (becomes a critical endpoint away from the chiral limit). Orange curve: position of the tricritical point as a function of the mass parameter $m$ (as compared to the other features displayed here, this last feature is rather sensitive to the degree of approximation with which we treat the equations).

[2] M. Tissier and N. Wschebor, Phys. Rev. D 84 (2011) 045018.

[3] D. Zwanziger, Nucl. Phys. B 323, 513 (1989).

[4] A. Cucchieri and T. Mendes, Phys. Rev. Lett. 100 (2008) 241601.

[5] M. Peláez, M. Tissier and N. Wschebor, Phys. Rev. D 88 (2013) 125003.

[6] J. A. Gracey, M. Peláez, U. Reinosa and M. Tissier, Phys. Rev. D 100, no. 3, 034023 (2019).

[7] J. I. Skullerud, P. O. Bowman, A. Kizilersu, D. B. Leinweber and A. G. Williams, JHEP 0304, 047 (2003).

[8] M. Peláez, U. Reinosa, J. Serreau, M. Tissier and N. Wschebor, Phys. Rev. D 96, no. 11, 114011 (2017).

[9] J. Braun, H. Gies and J. M. Pawlowski, Phys. Lett. B 684, 262 (2010).

[10] U. Reinosa, J. Serreau, M. Tissier and N. Wschebor, Phys. Rev. D 93, no. 10, 105002 (2016).

[11] T. K. Herbst, J. Luecker and J. M. Pawlowski, arXiv:1510.03830 [hep-ph].

[12] U. Reinosa, "Perturbative aspects of the deconfinement transition", Habilitation thesis, to appear.

[13] L. Fister and J. M. Pawlowski, Phys. Rev. D 88, 045010 (2013).

[14] M. Quandt and H. Reinhardt, Phys. Rev. D 94, no. 6, 065015 (2016).

[15] M. Fromm, J. Langelage, S. Lottini and O. Philipsen, JHEP 1201 (2012) 042.

[16] K. Kashiwa, R. D. Pisarski and V. V. Skokov, Phys. Rev. D 85 (2012) 114029.

[17] U. Reinosa, J. Serreau and M. Tissier, Phys. Rev. D 92, 025021 (2015).

[18] J. Maelger, U. Reinosa and J. Serreau, Phys. Rev. D 97074027 (2018). 
[19] K. Kashiwa and R. D. Pisarski, Phys. Rev. D 87 (2013) 096009.

[20] J. Maelger, U. Reinosa and J. Serreau, Phys. Rev. D 101, no. 1, 014028 (2020).

[21] R. D. Pisarski and F. Wilczek, Phys. Rev. D 29 (1984) 338.

[22] A. Bazavov, H.-T. Ding, P. Hegde, F. Karsch, E. Laermann, S. Mukherjee, P. Petreczky and C. Schmidt, Phys. Rev. D 95, no. 7, 074505 (2017). 